\title{
PRENATAL ALCOHOL EXPOSURE AND CORTICAL ANGIOGENESIS
}

Researchers at Normandy University, and Rouen and Brest Universities, France studied the effects of prenatal alcohol exposure on the cortical microvascular and the action of alcohol, glutamate, and vascular endothelial growth factor A (VEGF) on activity, plasticity, and survival of microvessels in mice. The prenatal alcohol effects on the cortical microvascular network in human controls and fetal alcohol syndrome (FAS)/partial FAS(pFAS) patients was also investigated at different developmental stages. In mice, prenatal alcohol exposure caused a reduction of cortical vascular density, loss of the radial orientation of microvessels, and altered expression of VEGF receptors. Glutamate-induced calcium mobilization in endothelial cells was inhibited, plasticity affected, and death of microvessels promoted. These effects of prenatal ethanol in mice were prevented by VEGF. In humans, a stage-dependent alteration of the vascular network was evident in the cortices of fetuses with pFAS/FAS. The radial organization of cortical microvessels was altered in pFAS/FAS patients from gestational week 30 to week 38 but not from week 20 to 22. Prenatal alcohol exposure affects cortical angiogenesis in mice and in pFAS/FAS patients. Vascular defects contribute to the brain abnormalities in fetal alcohol syndrome. (Jegou S, Ghazi FE, de Lendeu PK, et al. Prenatal alcohol exposure affects vasculature development in the neonatal brain. Ann Neurol 2012 Dec;72(6):952-60). (Resp.: Dr Gonzales. Bruno.gonzales@univ-rouen.fr).

COMMENT. Diagnostic characteristics of FAS include: 1) growth deficiency; 2) craniofacial abnormalities (smooth philtrum, thin upper lip, and small palpebral fissures); 3) CNS damage: structural-microcephaly, agenesis of corpus callosum, cerebellar hypoplasia; neurological-epilepsy, incoordination; and functional-cognitive and behavioral abnormalities (developmental delay, learning disabilities, ADHD); and 4) prenatal alcohol exposure. Associated abnormalities include ocular-strabismus, ptosis; cardiac; skeletal, and renal. Differential diagnosis includes Williams syndrome, Noonan syndrome, and fetal hydantoin syndrome. In addition to effects on the fetus, alcohol also has significant effects on the maternal vasculature, with alterations in blood flow in reproductive organs and placenta, and on angiogenesis. (Ramadoss J, Magness RR. Vascular effects of maternal alcohol consumption. Am J Physiol Heart Circ Physiol 2012 Aug 15;303(4):H414-21). Alcohol exposure affects brain development by numerous pathways and at all stages from neurogenesis to myelination. FAS is a brain disorder rather than primarily defined by physical characteristics. (Riley EP, et al. Neuropsychol Rev 2011 Jun;21(2):73-80).

The remarkably high prevalence of epilepsy and seizure history in fetal alcohol spectrum disorders (FASD) is reported from Queens University, ON, Canada. Of 425 subjects ages 2-49 attending two FASD clinics, 25 (5.9\%) had a diagnosis of epilepsy, and $50(11.8 \%)$ had at least one seizure episode, yielding a prevalence of seizures in 17.7\%. (Bell SH et al. Alcohol Clin Exp Res 2010 Jun;34(6):1084-9). 